\title{
Effectiveness of Right Median Nerve Electrical Stimulation in Improving Functional Status in Persons with Traumatic Brain Injury
}

\section{IJCRR}

Section: Healthcare

ISI Impact Factor

(2019-20): 1.628

IC Value (2019): 90.81

$\operatorname{SJIF}(2020)=7.893$

(c) (i) (8)

Copyright@IJCRR

\section{Divya Li ${ }^{1}$ Kumaresan A $^{2}$, Prathap Suganthirababu ${ }^{2}$, Vignesh $\mathbf{S}^{1}$}

'Tutor, Saveetha College of Physiotherapy, Saveetha Institute of Technical and Medical Sciences, Chennai-602105, Tamilnadu, India; ${ }^{2}$ Professor, Saveetha College of Physiotherapy, Saveetha Institute of Technical and Medical Sciences, Chennai-602105, Tamilnadu, India.

\section{ABSTRACT}

Background: Traumatic brain injury is one of the devastating clinical conditions, leading to loss of consciousness and arousal at some point of time after injury. The median nerve serves as the peripheral gateway for accessing the central nervous system among persons with coma.

Aim: The study aims to evaluate the effectiveness of the right median nerve electrical stimulation on functional status in participants with traumatic brain injury.

Methods: A quasi-experimental study was done on 30 traumatic brain injury participants. Participants were recruited after obtaining an informed consent form from the participant's caregiver. Ranchos Los Amigos Scale was used as a screening tool for the inclusion of participants. The pre-test measurement of functional status was obtained through the Disability rating scale. Right median nerve electrical stimulation was given over the right hand for four weeks. At the end of study duration, Post-test measurements were taken and data were tabulated and analyzed statistically by using Wilcoxon signed-rank test.

Results: It was observed that there was a significant improvement in functional status $(P<0.001)$ in participants with traumatic brain injury.

Conclusion: Right median nerve electrical stimulation is a safe, inexpensive, and noninvasive technique that has a beneficial effect on improving functional status among brain injury participants.

Key Words: Traumatic Brain Injury, Coma, Median Nerve, Right Median Nerve Stimulation, Functional Status, Disability Rating Scale

\section{INTRODUCTION}

Traumatic brain injury (TBI) is a form of acquired brain injury leading to insult to the brain due to sudden trauma and the Centers for Disease Control and Prevention (CDC) defines TBI as craniocerebral trauma related to neurologi$\mathrm{cal} /$ neuropsychological abnormalities, skull fracture, intracranial lesions or death. ${ }^{1,2}$ At the global, it has been estimated that the annual incidence and mortality from TBI are 200 and 20/100000/year, respectively. ${ }^{3,4}$ The incidence, mortality, and case-fatality rates in India were 150/100000, $20 / 100000$, and $10 \%$ based on an epidemiological study. ${ }^{4,5}$ In India, it is estimated that nearly about 1 million dies every year. ${ }^{6}$ Road Traffic accident (RTA) is regarded as one of the leading causes of TBI. RTA contributes to $(60 \%)$ of TBI followed by falls (20-25\%) and violence (10\%). ${ }^{4}$ Males are suspectable to TBI with $75 \%$ incidence in contrast to fe- males and the ratio of injury in male to female in India is about $3: 1 .^{7,8}$

TBI has been categorized into primary or secondary brain injury. The former is due to the consequences of the physical insult whose pattern and extent of damage depend upon the nature, intensity, and duration of impact. ${ }^{9}$ Primary and secondary TBI cause temporary and/or permanent dysfunction in the brain, which limits a patient's activities, affecting participation in society, and lowers the quality of life. ${ }^{10,11} \mathrm{About}$ $17 \%$ of TBI persons undergo a period of complete unconsciousness or coma. ${ }^{12} \mathrm{TBI}$ patients have a clinical presentation with the signs and symptoms of loss of consciousness, ear and nose bleed, seizures, paresis, nausea, balance deficits, cognitive communication and swallowing. ${ }^{13}$ Physical therapists play a vital role in the neurorehabilitation of traumatic brain injury patients.

\section{Corresponding Author:}

Kumaresan. A, Saveetha College of Physiotherapy, SIMTAS, Chennai-602105, Tamilnadu, India.

Email:kresh49@gmail.com

ISSN: 2231-2196 (Print)

Received: 19.01 .2021
ISSN: 0975-5241 (Online)

Revised: 24.02 .2021
Accepted: 09.03.2021 
The disorders of consciousness (DOC) include coma, vegetative state, and minimally conscious state. Clinical diagnosis of coma is defined by absent or limited vocal or muscle activity and presence of decreased or any abnormal response to noxious stimuli, an absence of sleep-wake cycle. ${ }^{14}$

Severe DOC range from coma via vegetative state (VS) also referred to as unresponsiveness wakefulness syndrome, a condition during which the patient shows no behavioural evidence of conscious-to minimally conscious state (MCS), characterized by one or more minimal but definite behavioural signs of consciousness. ${ }^{15,18}$

In the past decades, physiotherapy techniques including neuromuscular electrical stimulation have played a vital role in neurorehabilitation for facilitating or inhibiting muscle tone. It also plays an essential role in unconscious patients. For providing stimulation, the median nerve of the right arm was selected. The right side of the brain has a major representation for cortical reorganization and is responsible for cognitive and sensory reorganization. The sensory distribution of the right hand has a larger cortical representation in the brain. Median nerve stimulation might function by acting on a few neurotransmitters. ${ }^{19,20}$ The disability rating scales (DRS) has been utilized in moderate and severe TBI. DRS can be easily administered and has good reliability and validity. This study aims to evaluate the effect of the right median nerve stimulation on functional status among traumatic brain injury participants.

\section{Methods}

The study was a Quasi-experimental study conducted in Saveetha Medical Hospital, Thandalam, Chennai. The sample was drawn from the Physiotherapy neurorehabilitation centre and Inpatient Units at Saveetha Medical Hospital. The study was conducted after getting approval from the Institutional Human Ethical Committee of Saveetha Institute of Medical and Technical Sciences 038/07/2020/IRB-HS/ SIMATIC. A convenient sampling technique was used for 30 participants in the study. The safety and simplicity of the procedure were explained to participants caregivers and an informed consent form was obtained from them. Ranchos Los Amigos (RLA) scale was used as a confirmatory test for the inclusion of participants. Selection criteria included RLA Level 1-2 participants, both male, and female, aged between 20 to 55 years, hand dominance-right hand. Participants were excluded if there was, vital signs of unstable, injury of median nerve/brachial plexus and fracture of right wrist, metallic implant in the right upper limb, cervical spinal cord injury, absent sensation in the thenar eminence of the right hand, history of seizures, Cardiac arrhythmia /Pacemaker implanted and pregnancy.

Pre-treatment reading of functional status was noted by using a disability rating scale. After the pre-test measures, par- ticipants received treatment through the right side median nerve stimulation. The participant position was supine lying with upper extremity elbow extended, forearm supinated, wrist and fingers extended. The right side forearm was selected for treatment and Neuromuscular electrical stimulation equipment was used.

In order, to reduce the skin resistance hand was wiped with a cotton swab. The electrode placement was active electrode over the volar aspect of the right forearm and the inactive electrode over the volar $2 / 3^{\text {rd }}$ of the right forearm. Parameters of neuromuscular electrical stimulation- the type of current: surged faradic current, waveform: asymmetric biphasic, frequency: 40hz, duration: $300 \mathrm{~ms}$, amplitude: $20 \mathrm{sec} /$ min, no of contractions: based on the response of muscle to avoid fatigue. The skin was examined for erythema under the electrode site during each treatment session. Similarly, Post-treatment readings were noted by using a disability rating scale.

\section{RESULTS}

The collected data were tabulated and analyzed using a nonparametric test. Wilcoxon signed-rank test was utilized for within-group analysis. The significance level of $(\mathrm{P}<0.001)$ was considered statistically significant. The results are presented in Table 1. There was a significant difference in pretest and post-test values of DRS $(\mathrm{P}<0.001)$.

As per literature, right median nerve stimulation has been reported to improve the outcomes for traumatic comatose cases. ${ }^{21}$ The study was done to investigate the effect of the right median nerve stimulation on the functional status by utilizing DRS. DRS scale ranges from 0 to $29 .{ }^{22}$ The lowest score represents no disability and the highest score is 29 that represents an extreme vegetative state. In the present study from Table 1, the pre-test median score was 28 and after the treatment, the post-test median score was observed to be 24 within the group. The $\mathrm{Z}$ value $=4.316$ and $\mathrm{P}<0.001$ denotes statistical improvement within the group.

\section{DISCUSSION}

Hence, the right side median nerve stimulation has effective recovery on functional recovery among traumatic brain injury participants and the probable reason for improvement might be activation of ascending reticular activating system(ARAS) through afferent stimuli provided via right median nerve stimulation and the spinoreticular component of the median nerve synapsing with the neurons of the ARAS. ${ }^{23}$ A study conducted by Kwan et al. have stated that when the right median nerve is stimulated with certain painful intensity, it results in activation of ARAS and serve as the 
pathway for the therapeutic function of electrical stimulation. $^{24}$

Over, a period of four weeks of study duration, the results of the present study revealed that there was significant functional recovery among traumatic brain injuries by the administration of the right median nerve stimulation. The limitation of the study includes the recruitment of participants from a single study centre and qualitative outcomes were used. In the future, it is recommended that the same study can be done with a large sample population based on age, the severity of the injury and quantitative outcomes such as EEG, fMRI, SPECT scans can be used to assess the cortical reorganization.

\section{CONCLUSION}

The study concludes that the right median nerve has a beneficial effect on the functional status of traumatic brain injury participants. Right median nerve stimulation is easy to administer, inexpensive and can be used in neurorehabilitation in participants with decreased consciousness.

\section{ACKNOWLEDGEMENT}

The author acknowledges the support received from the Department of Saveetha College of Physiotherapy, Chennai. Authors acknowledge the authors/editors/publishers of all those articles, journals and books that have been utilized in the study.

\section{Funding Source: Self}

\section{Conflict of Interest: Nil}

\section{REFERENCES}

1. Solmaz et al. Traumatic brain injury due to gunshot wounds. A single Institution's experience with442 consecutive patients. Turk Neurosurg.2009;19:216-23.

2. Centers for Disease Control and Prevention (CDC). National centre for traumatic brain injury prevention and control.Report to Congress on mild traumatic brain injury in the United States: Steps to prevents serious public health problem.Atlanta (GA): Centers for Disease Control and Prevention. 2003.

3. Romer CJ, Zitnay G, Gururaj G et al. Prevention, critical care and rehabilitation of Neurotrauma WHO Collaborating centre for Neurotrauma.1995.

4. Gururaj G. An Epidemiological approach to prevention, prehospital care and Rehabilitation In neurotrauma,Neurology India.1995;43(3):106.

5. Gururaj G. Epidemiology of Traumatic brain injuries.Indian Scenario. Neur Res.2002;24:1-5.

6. Masel B, Dewitt D. Traumatic Brain injury disease.Long term consequences of traumatic brain injury. In H. Levin, D\&R.
Chan(Eds), Understanding traumatic brain injury. Curr Res Fut Direct.2014.

7. Iranmanesh.F. Outcome of Head trauma. Indian J Pediatr.2009;76(9):929-31.

8. Oyedele EA, Andy E, Solomon GM, Rifkatu L, Nanbur S. The prevalence of traumatic the head is seen in a tertiary health facility in North-Central Nigeria.IJPHR.2015;3(4):127-9.

9. Mass AI MAGS.Prognosis and clinical trial design in traumatic brain injury: The IMPACT study.2007.

10. B.T Mausbach, et al. Activity Restriction and depression in medical patients and their caregivers. A meta-analysis, ClinicalPsychology Review.2011; Vol 3: No 6,pp.900-908.

11. K.A Cappa, J.C Conger, A.J. Conger. Injury severity and outcome. A meta-analysis of prospective studies on TBI. Health Pschol. 2011;30(5): 542-560.

12. Mohammad Ali Heidari Gorijiet al. Effect of auditory Stimulation on traumatic coma duration in intensive care unit of the Medical Sciences University of Mazandaran. 2014;8(1);69-72.

13. Gill P, Gill T, Kamath A, Whisnant B. Readability assessment of concussion and traumatic brain injury publications by the Centers for Disease Control and Prevention. Int J General Med. 2012; 5:923-933.

14. Laureys S, Owen A, Schiff N. Brain Function in a coma, vegetative state, and related disorders. Lancet Neur.2004;3(9):537-46.

15. Jennett B, Plum F. Persistent Vegetative state after brain damage- A syndrome in search of a name. The Lancet.1972;229(7753):734-37.

16. LaureysS,Celesia G G,Cohadon F, Lavrijsen J , Leon-Carrion J, Sannita WG et al. European Task Force on Disorders of consciousness Unresponsive wakefulness syndrome: A new name for the vegetative state or apallic syndrome. BMC Med.2010;868.

17. Giacinto JT, Ashwal S, Childs N, Cranford R, Jennett B, Katz DI et al. The minimally conscious state: definition and diagnostic criteria. Neurology.2002; 58(3):349-53.

18. Giacino JT, Kalmar K, Whyte J. The JFK Coma recovery scaleRevised measurement characteristics and diagnostic utility. Arch Phys Med Rehabil.2004;85:2020-2029.

19. Cooper EB, Scherder EJ, Cooper JB. Electrical treatment of reduced consciousness: experience with coma and Alzheimer's disease. Neuropsychol Rehabil. 2005;15:389-405.

20. Lei J, Wang L Gao G, Cooper E, Jiang J. Right Median Nerve Electrical Stimulation for acute traumatic coma patients. J Neurotrauma.2015;32(20):1584-1589.

21. Michal VaimanEphraimnEviatar, Samuel Segal, Tel Aviv, Israel, Surface electromyographic studies of swallowing in normal subjects. A review of 440 adults. Report Quantitative Data. Timing Measures Otolaryngology-Head and Neck Surgery. 2004;131:548-555.

22. Rappaport M, Hall KM, Hopkins K, Belleza T, Cope DN. A disability rating scale for severe head trauma: coma to community. Arch Phys Med Rehabil.1982;63(3):118-23.

23. Kaur H, Gupta D, Sharma V. Right Median nerve stimulation for improving consciousness: A case series.Int J Neo Ther. 2015;12(2):144-8.

24. Kwan CL, Crawley AP, Mikulis DJ, Davis KD. An fMRI study of the anterior cingulated cortex and surrounding medial wall activations by noxious cutaneous heat and cold stimuli. Pain.2000;85(3):359-74. 
Table 1: Pre-Test and Post-test of Disability Rating Scale

\begin{tabular}{|c|c|c|c|c|}
\hline Disability rating scale & $\mathbf{N}$ & Median & $\begin{array}{c}\text { W+ value } \\
\text { T value }\end{array}$ & P-value \\
\hline $\begin{array}{l}\text { Pre-test } \\
\text { Post-test }\end{array}$ & 30 & 28 & $\begin{array}{c}\mathrm{W}=-300.0 \\
\mathrm{~T}+=0.00 \\
\mathrm{~T}-=-300.0 \\
\text { Z Value }=-4.316\end{array}$ & $\mathrm{P}=<0.001$ \\
\hline
\end{tabular}

